\title{
Factores de riesgo de presentar una complicación postoperatoria en pacientes operados de urgencia por patología herniaria*
}

\author{
Dr. FRANCISCO VENTURELLI M..$^{1,2,3}$ \\ 1 Universidad Austral de Chile. Campo Clínico Osorno. \\ 2 Servicio de Cirugía Urgencia. Hospital Base Osorno. \\ 3 Servicio de Cirugía. Hospital Base Osorno.
}

\section{TRABAJO DE INGRESO}

\begin{abstract}
Risk factors for complications and mortality after emergency surgery for complicated hernias

Background: Thirteen percent of hernias require emergency surgery and of these, approximately $14 \%$ require an intestinal excision. Aim: To identify risk factors for postoperative complications after emergency surgical treatment of complicated hernias. Material and Methods: Using surgical room registries, all patients subjected to emergency surgery for complicated hernias between 2004 and 2008 were identified and their medical records were reviewed. Follow up was performed using data from hospital and outpatient medical records. Results: One hundred fifty two patients were identified but four were discarded due to lack of complete records. Therefore, 148 patients aged 24 to 95 years (104 females) were analyzed. Forty patients had postoperative complications (27\%) and six died (4\%). Obesity, hypertension, diabetes mellitus and an American Society of Anesthesiologists (ASA) classification, III or IV, were identified as risk factors for complications. Logistic regression only accepted hypertension as a risk factor with an odds ratio (OR) of 2.35 (95\% confidence intervals (CI) 1.04-5.3). The predictors for mortality were obesity, hypertension, an ASA classification of III or IV and having a strangulated hernia. Logistic regression only accepted having a strangulated hernia as an independent risk factor with an OR of 16.4 (95\% CI 1.6-167.7). Conclusions: Hypertension and having a strangulated hernia are risk factors for complications and mortality after emergency surgery for complicated hernias.
\end{abstract}

Key words: Complicated hernia, emergency surgery, complications.

\section{Resumen}

Introducción: Obteniendo los factores de riesgo de morbimortalidad postoperatoria, al tratar una hernia complicada de urgencia, podríamos definir mejor su tratamiento. Objetivo principal: Identificar los factores de riesgo de morbilidad postoperatoria en pacientes operados de urgencia por una hernia complicada en el Hospital Base de Osorno (HBO). Material y Método: Cohorte retrospectiva. Se incluyeron pacientes mayores de 14 años operados por patología herniaria complicada en el servicio de urgencia del HBO entre los

*Recibido el 12 de Septiembre de 2010 y aceptado para publicación el 10 de Octubre de 2010.

Correspondencia: Dr. Francisco Venturelli M.

Guillermo Buhler 1645 Depto. 504, Osorno, Chile.

fcoventurelli@gmail.com 
años 2004 y 2008. Se excluyeron aquellos que no contaron con datos en las variables de interés. Se realizó un análisis bivariado y una regresión logística. Medida de riesgo: odds ratio (OR). Intervalo de confianza 95\%. Stata 10.0. Resultados: La cohorte incluyó 148 pacientes. Mediana de edad 66 años (24-95). La morbilidad y mortalidad postoperatoria fue un $27,02 \%$ (40 pacientes) y $4,05 \%$ (6 pacientes) respectivamente. Las variables obesidad, hipertensión arterial (HTA), diabetes mellitus (DM) y el tener un ASA III-IV resultaron ser significativas $(\mathrm{p}<0,05)$ para morbilidad postoperatoria en el análisis bivariado. En la regresión logística, sólo el ser hipertenso resultó ser un factor de riesgo (OR: 2,35, IC 95\%: 1,04-5,30). Los factores de riesgo para mortalidad que resultaron significativos en el análisis bivariado fueron ser obeso, hipertenso, presentar un ASA de III-IV y presentar hernia estrangulada $(\mathrm{p}<0,05)$. En el análisis multivariado sólo el presentar una hernia estrangulada fue un factor de riesgo (OR: 16,4, IC 95\%: 1,59-167,76). Conclusión: Ser hipertenso y la presencia de necrosis en el saco herniario son factores de morbilidad y mortalidad postoperatoria respectivamente en el paciente que se opera por una hernia complicada de urgencia.

Palabras clave: Hernia abdominal, morbilidad, mortalidad, factores de riesgo, incarceración.

\section{Introducción}

La hernia de pared abdominal es una patología muy frecuente. De no tratarse en el momento adecuado puede complicarse mediante un atascamiento o estrangulamiento ${ }^{1}$. Hasta el $13 \%$ de las hernias requieren de resolución quirúrgica de urgencia y de éstas, el 13-15\% requieren resección intestinal ${ }^{2,3}$.

La instalación de prótesis en la cirugía herniaria disminuye la recidiva en forma importante ${ }^{4,5}$. Sin embargo, su uso en la cirugía de urgencia es aún escaso, principalmente por el temor a complicaciones asociadas a ésta, aumentando la morbilidad del paciente.

En los últimos años, hemos visto como ha aumentado el uso de los materiales protésicos tanto en hernias atascadas como estranguladas, con resultados alentadores ${ }^{6-8}$. Al enfrentar a uno de estos pacientes, sería importante identificar cuáles son los factores de riesgo reales de que aparezca una complicación al tratar una hernia complicada de urgencia, para así poder definir con mayor exactitud el riesgo de cada paciente y en base a esto definir el uso o no de prótesis según cada caso.

El objetivo principal de este estudio, es identificar cuáles son los factores de riesgo de presentar una complicación postoperatoria en pacientes operados de urgencia por una hernia complicada en el Hospital Base de Osorno. El objetivo secundario es identificar cuáles son los factores de riesgo de mortalidad postoperatoria.

\section{Material y Método}

Diseño: Cohorte retrospectiva. Por medio del registro de pabellón, se buscan todos los pacientes operados por patología herniaria en el servicio de urgencia del Hospital Base Osorno (HBO) entre los años 2004 y 2008 para luego buscar las variables de interés en la ficha clínica correspondiente.

Población: Se incluyeron todos los pacientes con edad mayor a 14 años ingresados al servicio de urgencia del HBO con diagnóstico de hernia umbilical, inguinal, crural, epigástrica o incisional complicada, definida como:

Hernia irreductible de menos de cinco días de evolución que se asocie a:

a) Signos de obstrucción intestinal: dolor + distensión abdominal + vómitos de retención y/o ausencia de eliminación de gases por el ano en las últimas $24 \mathrm{~h}$, y/o salida a líquido de retención por sonda nasogástrica (SNG) y radiografía abdomen simple que muestre asas dilatadas con o sin niveles.

b) Signos de isquemia intestinal: dolor + signos de obstrucción intestinal + signos de irritación peritoneal o cambio de la coloración de la piel que cubre el saco, o compromiso hemodinámico.

c) Signos de atascamiento o necrosis de epiplón: hernia irreductible con o sin cambio de coloración de la piel asociada a dolor intenso a la palpación de ella sin evidencias de compromiso intestinal.

Se excluyeron a todos los pacientes que no contaron con datos en las variables de interés.

Todo paciente de la población de estudio fue intervenido por un cirujano del servicio de urgencia del hospital, durante el período intraoperatorio, este decidió instalar o no una prótesis de polipropileno, (hernioplasía con malla o herniorrafia respectivamente), dependiendo de su propia decisión. A todo paciente se le indicó profilaxis antibiótica preoperatoria contra Gram negativos y anaerobios.

El seguimiento se realizó mediante los datos que aparecían en las fichas clínicas en el tiempo de su estadía hospitalaria y posterior al alta en los controles 
por cirujano en el policlínico de nuestro hospital. La fecha de término del seguimiento fue el 2010.

Variables de interés: Todas las variables fueron recogidas por medio de la revisión de fichas clínicas. Variables dependientes:

1. Complicación postoperatoria: infección de la herida operatoria, seroma de la herida operatoria, retiro parcial o total de la malla, otra complicación (neumonía, trombosis mesentérica, obstrucción intestinal, hematoma de herida operatoria que requirió de drenaje quirúrgico, shock séptico, perforación intestinal, necrosis cutánea), recidiva herniaria (esta no fue incluida dentro de la morbilidad posoperatoria para análisis bivariado y multivariado)

2. Mortalidad postoperatoria, que se definió como muerte antes de los 30 días posoperatorios para los que permanecieron menos de 1 mes hospitalizados y muerte intrahospitalaria para los que permanecieron hospitalizados más allá de este período.

Variables independientes recopiladas fueron: Edad ( $<65$ años o $>=65$ años), sexo, ubicación de la hernia (inguinal, crural, umbilical, epigástrica, incisional), DM, HTA, limitación crónica del flujo aéreo (LCFA), insuficiencia cardiaca (IC), obesidad, insuficiencia renal crónica (IRC), accidente vascular encefálico (AVE), otra comorbildad (daño hepático crónico, mesenquimopatía, coronariopatía), ASA (I-II o III-IV), presencia de estrangulamiento del contenido herniario (necesidad de resección de intestino necrótico y/o de epiplón necrótico), Instalación de material protésico, instalación de drenaje, tipo de anestesia (raquídea o general).

Análisis estadístico: Al tener las variables de interés se realizó un análisis bivariado mediante $\mathrm{Chi}^{2}$ o test de Fisher según corresponda, para luego hacer un análisis multivariado mediante regresión logística con las variables que resulten con significación estadística en el primer análisis. La medida de riesgo obtenida será odds ratio (OR) y su intervalo de confianza será de un $95 \%$. El programa estadístico utilizado fue el Stata 10.0

\section{Resultados}

En total, se recopilaron 152 pacientes, de los cuales 4 fueron eliminados por no tener datos en las variables de análisis, quedando 148 para el estudio.

La mediana de edad fue 66 años (24-95), el sexo predominante fue el femenino con 104 (70,27\%) casos. La ubicación herniaria más frecuente en las mujeres fue la incisional con $41(39,42 \%)$ casos y la inguinal en los hombres con $25(56,81 \%)$ casos. La distribución de las hernias según localización fue: $45(30,40 \%)$ incisionales, $33(22,29 \%)$ inguinales, $31(20,94 \%)$ umbilicales, $30(20,27 \%)$ crurales y 9 $(6,08 \%)$ epigástricas.

Del total de la cohorte, $59(39,86 \%)$ pacientes presentaban antecedentes de comorbilidad asociada al momento del diagnóstico.

Cincuenta y seis $(37,83 \%)$ presentaban HTA, 24 (16,21\%) diabetes mellitus, 21 (14,18\%) obesidad, $18(12,16 \%)$ limitación crónica al flujo aéreo, 10 $(6,75 \%)$ insuficiencia cardiaca, $6(4,05 \%)$ insuficiencia renal crónica, $5(3,37 \%)$ daño hepático crónico, 3 $(2,02 \%)$ coronariopatía, $2(1,35 \%)$ accidente vascular encefálico y $2(1,35 \%)$ mesenquimopatía.

A $120(81,08 \%)$ pacientes se les diagnosticó una hernia atascada, de ellas $38(31,66 \%)$ fueron reparadas con malla. De las hernias estranguladas, 28 $(18,91 \%)$ casos, en $3(10,71 \%)$ se instaló material protésico. La mediana de días postoperatorios fue 4 (1-70) días. La mediana de seguimiento fue 37 (2-67) meses.

La morbilidad postoperatoria fue un $27,02 \%$ (40 pacientes), 39,28\% (11 pacientes) en las hernias estranguladas y $24,16 \%$ (29 pacientes) en las atascadas. Entre las complicaciones quirúrgicas, las más frecuentes fueron la infección de herida operatoria y la recidiva herniaria con $21(14,18 \%)$ casos cada uno (Tabla 1). La mediana de seguimiento fue de 38 (10-67) meses.

La mortalidad postoperatoria fue de 4,05\% (6 pacientes), $2(1,35 \%)$ por peritonitis y $4(2,79 \%)$ por shock séptico refractario.

Las variables obesidad, HTA, DM y el tener un ASA III-IV resultaron ser significativas $(\mathrm{p}<0,05)$ para morbilidad postoperatoria (Tablas 2 y 3 ). Al realizar la regresión logística con estas variables, sólo el ser hipertenso resultó ser un factor de riesgo (OR: 2,35, IC95\%: 1,04-5,30) (Tabla 4).

Tabla 1. Morbilidad postoperatoria en los pacientes operados de urgencia por una hernia de pared abdominal complicada $(n=148)$

\begin{tabular}{lcc}
\hline Morbilidad postoperatoria & n & $(\mathbf{( \% )}$ \\
\hline Infección de herida operatoria & 21 & $(14,18 \%)$ \\
Recidiva herniaria & 21 & $(14,18 \%)$ \\
Seroma & 13 & $(8,78 \%)$ \\
Shock séptico & 5 & $(3,37 \%)$ \\
Peritonitis & 2 & $(1,35 \%)$ \\
Hematoma herida operatoria & 2 & $(1,35 \%)$ \\
Neumonía & 1 & $(0,67 \%)$ \\
Retiro de malla & 1 & $(2,43)^{*}$ \\
\hline
\end{tabular}

*porcentaje del total de hernias operadas con malla. 
FACTORES DE RIESGO DE PRESENTAR UNA COMPLICACIÓN POSTOPERATORIA EN PACIENTES OPERADOS DE...

Tabla 2. Análisis bivariado de factores de riesgo de morbilidad y mortalidad postoperatoria en los pacientes operados de urgencia por una hernia de pared abdominal complicada

\begin{tabular}{|c|c|c|c|c|c|c|}
\hline Variable & $\begin{array}{l}\text { Morbilidad } \\
\text { presente (\%) }\end{array}$ & $\begin{array}{l}\text { Morbilidad } \\
\text { ausente (\%) }\end{array}$ & $\mathbf{p}$ & $\begin{array}{c}\text { Mortalidad } \\
\text { presente (\%) }\end{array}$ & $\begin{array}{l}\text { Mortalidad } \\
\text { ausente (\%) }\end{array}$ & $\mathbf{p}$ \\
\hline \multicolumn{7}{|l|}{ Edad } \\
\hline$>65$ años & $21(14,19)$ & $56(37,84)$ & \multirow[t]{2}{*}{0,506} & $5(3,37)$ & $72(48,64)$ & \multirow[t]{2}{*}{0,125} \\
\hline$<65$ años & $16(10,81)$ & $55(37,16)$ & & $1(0,67)$ & $70(47,29)$ & \\
\hline \multicolumn{7}{|l|}{ Sexo } \\
\hline Masculino & $9(6,08)$ & $35(23,65)$ & \multirow[t]{2}{*}{0,406} & $6(4,05)$ & $98(66,21)$ & \multirow[t]{2}{*}{0,115} \\
\hline Femenino & $28(18,92)$ & $76(51,35)$ & & $0(0)$ & $44(29,72)$ & \\
\hline \multicolumn{7}{|l|}{ LCFA } \\
\hline Presente & $6(4,05)$ & $12(8,11)$ & \multirow[t]{2}{*}{0,384} & $1(0,67)$ & $17(11,48)$ & \multirow[t]{2}{*}{0,547} \\
\hline Ausente & $31(20,95)$ & $99(66,89)$ & & $5(3,37)$ & $25(16,89)$ & \\
\hline \multicolumn{7}{|l|}{ Obesidad } \\
\hline Presente & $10(6,76)$ & $11(7,43)$ & \multirow[t]{2}{*}{0,010} & $3(2,02)$ & $18(12,16)$ & \multirow[t]{2}{*}{0,037} \\
\hline Ausente & $27(18,24)$ & $100(67,57)$ & & $3(2,02)$ & $124(83,78)$ & \\
\hline \multicolumn{7}{|c|}{ Hipertensión arterial } \\
\hline Presente & $21(14,19)$ & $35(23,65)$ & \multirow[t]{2}{*}{0,006} & $5(3,37)$ & $51(34,45)$ & \multirow[t]{2}{*}{0,019} \\
\hline Ausente & $16(10,81)$ & $76(51,35)$ & & $1(0,67)$ & $91(61,48)$ & \\
\hline \multicolumn{7}{|c|}{ Insuficiencia cardíaca } \\
\hline Presente & $4 \quad(2,70)$ & $6(4,05)$ & \multirow[t]{2}{*}{0,218} & $0(0)$ & $10(6,75)$ & \multirow[t]{2}{*}{0,652} \\
\hline Ausente & $33(22,30)$ & $105(70,95)$ & & $6(4,05)$ & $132(89,18)$ & \\
\hline \multicolumn{6}{|l|}{ IRC } & \multirow[t]{3}{*}{0,223} \\
\hline Presente & $3(2,03)$ & $3(2,03)$ & 0,165 & $1(0,67)$ & $5 \quad(3,37)$ & \\
\hline Ausente & $34(22,97)$ & $108(72,97)$ & & $5(3,37)$ & $137(92,56)$ & \\
\hline \multicolumn{7}{|l|}{ AVE } \\
\hline Presente & $0 \quad(0)$ & $2(1,35)$ & \multirow[t]{2}{*}{0,561} & $0(0)$ & $2(1,35)$ & \multirow[t]{2}{*}{0,920} \\
\hline Ausente & $37(25)$ & $109(73,65)$ & & $6(4,05)$ & $140(94,59)$ & \\
\hline \multicolumn{7}{|c|}{ Diabetes Mellitus II } \\
\hline Presente & $10(6,76)$ & $14(9,46)$ & \multirow[t]{2}{*}{0,039} & $3(2,02)$ & $21(14,18)$ & \multirow[t]{2}{*}{0,054} \\
\hline Ausente & $27(18,24)$ & $97(65,54)$ & & $3(2,02)$ & $121(81,75)$ & \\
\hline
\end{tabular}

*LCFA limitación crónica del flujo aéreo; IRC: insuficiencia renal crónica; AVE: accidente vascular encefálico.

Para la mortalidad, los factores de riesgo que resultaron significativos en el análisis bivariado fueron ser obeso, hipertenso, presentar un ASA de III-IV $y$ tener una hernia estrangulada $(\mathrm{p}<0,05)$ (Tablas 2 y 3). En el análisis multivariado, sólo el presentar una hernia estrangulada fue un factor de riesgo (OR: 16,4, IC 95\%: 1,59-167,76) (Tabla 4).

\section{Discusión}

La hernioplastía con malla es el tratamiento de elección para las hernias operadas en forma electiva $^{9}$. Sin embargo, en nuestra realidad, hay muchos pacientes que no se alcanzan a operar por esta vía y llegan a consultar a los servicios de urgencia cuando la hernia se atasca o estrangula. Lamentablemente la mayor parte de la información existente sobre pato- logía herniaria, es en base a cirugías electivas, haciendo difícil la toma de decisiones cuando se trata de una hernia complicada. Actualmente, en nuestros servicios de urgencia, es común encontrarnos con esta patología, por lo que es trascendental para el cirujano de urgencia conocer más sobre ella.

Uno de los mayores temores al enfrentar a un paciente con una hernia atascada o estrangulada es la morbimortalidad postoperatoria y la factibilidad de reparar la pared abdominal. Varios son los factores de riesgo que se han asociado a la morbimortalidad, por ejemplo la edad, la comorbilidad, la presencia de necrosis intestinal, etc. Sin embargo, varios estudios al respecto se basan en análisis bivariado disminuyendo con eso la validez de los resultados ${ }^{2,10,11}$.

La mediana de edad de estos pacientes en todas las publicaciones, es bastante alta ${ }^{6,10,11}$. Esta serie alcanzó los 66 años (24-95), lo que hace que el 
F. VENTURELLI M.

Tabla 3. Análisis bivariado de factores de riesgo de morbilidad y mortalidad postoperatoria en los pacientes operados de urgencia por una hernia de pared abdominal complicada

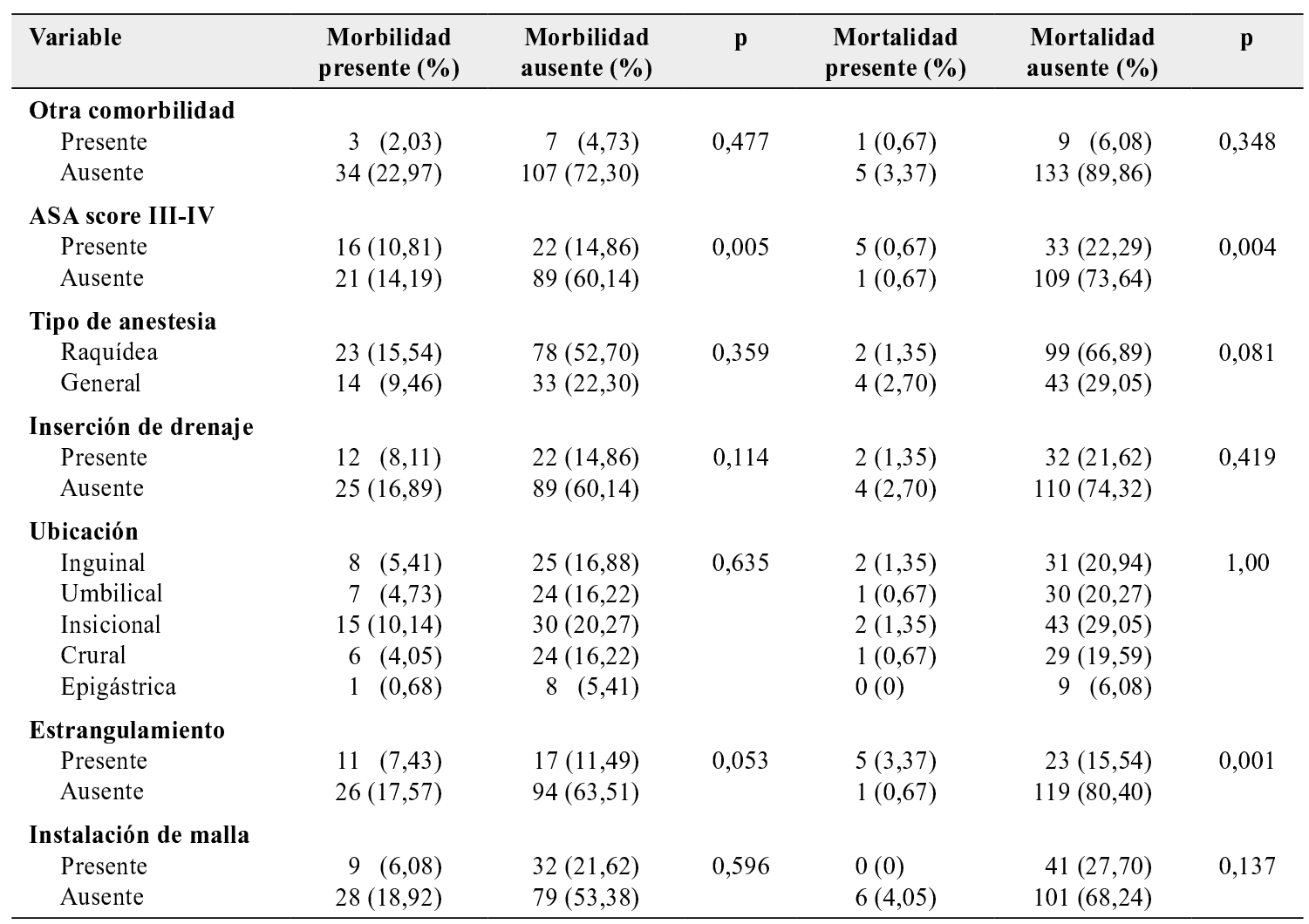

Tabla 4. Análisis multivariado de los factores de riesgo para morbilidad y mortalidad postoperatoria en los pacientes operados de urgencia por una hernia de pared abdominal complicada

\begin{tabular}{lccclcccc}
\hline Variable & & Morbilidad & \multicolumn{3}{c}{ Variable } & \multicolumn{3}{c}{ Mortalidad } \\
& OR & $\mathbf{9 5 \%}$ CI & $\mathbf{p}$ & & OR & $\mathbf{9 5 \%}$ CI & p \\
\hline Diabetes Mellitus II & 2,18 & $0,82-5,80$ & 0,116 & HTA & 7,88 & $0,68-91,43$ & 0,098 \\
Obesidad & 2,01 & $0,69-5,82$ & 0,197 & ASA Score III-IV & 6,56 & $0,58-$ & 73,17 & 0,126 \\
Hipertensión arterial & 2,35 & $1,04-5,30$ & 0,039 & Estrangulación herniaria & 16,34 & $1,59-167,76$ & 0,019 \\
ASA Score III-IV & 2,26 & $0,94-5,40$ & 0,066 & Obesidad & 1,79 & $0,21-$ & 15,26 & 0,59 \\
\hline
\end{tabular}

porcentaje de patologías asociadas sea también alto (39,86\% en nuestro estudio).

A diferencia de otros trabajos, la mayoría de nuestros pacientes fueron del sexo femenino con $104(70,27 \%)$ casos $^{8,12}$. En el hombre la ubicación herniaria más frecuente fue la inguinal. En la mujer, a diferencia de la literatura, fue la incisional ${ }^{2,13}$.

La gran mayoría de los pacientes de la cohorte expuesta presentaron hernias atascadas (120 pacien- tes), siendo las hernias estranguladas un $18 \%$. Este último porcentaje es levemente superior a lo publicado por otras series que reportan cifras de hasta un $15 \%$. 2,3 . Esta situación se explica por la edad y la alta ruralidad de muchos de nuestros pacientes lo que los hace consultar tardíamente.

En cuanto al análisis bivariado y multivariado, las distintas comorbilidades se analizaron por separado pensando en el hecho de que algunas enfermedades 
se asocian más a infección que otras, por ejemplo, la diabetes mellitus ${ }^{14}$.

En el análisis bivariado, para morbilidad se obtuvo que la obesidad, la HTA, la diabetes mellitus y el ASA III-IV fueron estadísticamente significativos $(\mathrm{p}<0,05)$ pero sólo la HTA demostró ser un factor de riesgo (OR: 2,35, IC95\%: 1,04-5,30). Hay estudios que demuestran que estos factores sí se asocian a morbilidad postoperatoria, pero varios están basados en análisis bivariado. Alvarez et $\mathrm{al}^{10}$, muestran en su estudio que el ASA III y IV y el presentar comorbilidad se asocia a una mayor morbimortalidad mediante este tipo de análisis, lo que concuerda con este estudio, pero no resultan ser factores de riesgo al realizar la regresión logística. Kuhla et $\mathrm{al}^{2}$, demuestran que la edad avanzada y la comorbilidad se asocian a una mayor mortalidad, pero tampoco hace un análisis multivariado.

En el estudio de Derici et $\mathrm{al}^{12}$, encontraron que el presentar una enfermedad asociada, el tener un ASA III o IV, el tipo de anestesia, la presencia de estrangulación herniaria y la presencia de necrosis se asocian a una mayor morbilidad en el análisis bivariado, pero sólo la presencia de necrosis resultó ser un factor de riesgo (OR: 4,52, IC 95\%: 1,59-12,84). Nuestro estudio concuerda con este último en que la gran mayoría de estas variables no son factores de riesgo de morbilidad. La presencia de necrosis, como variable única, en el presente estudio, no se asoció como factor de morbilidad $(\mathrm{p}=0,053)$.

La instalación de malla, al igual que en otras publicaciones, no tuvo ninguna implicancia en la morbimortalidad postoperatoria ${ }^{3,6-8,12,15-18}$. Si bien una de las mallas tuvo que ser retirada por infección, esta complicación no aumentó el riesgo de morbilidad. Por otra parte, esta infección se produjo en un paciente con una hernia atascada (sin presencia de necrosis), por lo que talvez en este paciente influyeron otros factores, no detectados, que incidieron en esta complicación y no la sola contaminación del sitio operatorio. Si bien este estudio nos sugiere que el instalar una malla no sería un factor de riesgo de morbilidad postoperatoria, el diseño ideal para evaluar este punto es un ensayo clínico, por lo que hay que tomar este resultado con cautela.

En el análisis de los posibles factores de riesgo de mortalidad, encontramos que la presencia de estrangulamiento fue la única variable que resultó actuar como tal (OR: 16,4, IC 95\%: 1,59-167,76), lo cual concuerda con el trabajo de Derici et al ${ }^{12}$. Este factor parece ser hasta ahora el más importante dentro de la morbimortalidad postoperatoria de la patología herniaria de urgencia.

Probablemente los grupos que presentan menos factores de riesgo, según los resultados del presente estudio, serían los más adecuados para iniciar una experiencia de reparación con prótesis en el futuro.

Como conclusión podemos decir que el presentar hipertensión arterial y la presencia de necrosis en el saco herniario son factores de riesgo, asociados a mayor morbilidad y mortalidad postoperatoria respectivamente, en el paciente que se opera por una hernia complicada de urgencia.

\section{Referencias}

1. O'Dwyer PJ, Norrie J, Alani A, Walker A, Duffy F, Horgan P. Observation or operation for patients with an asymptomatic inguinal hernia: a randomized clinical trial. Ann Surg. 2006;244:167-73.

2. Kulah B, Kulacoglu I, Oruc M, Duzgun A, Moran M, Ozmen M, et al. Presentation and outcome of incarcerated external hernias in adults. Am J Surg. 2001;181:1014.

3. Kurt N, Oncel M, Ozkan Z, Bingul S. Risk and outcome of bowel resection in patients with incarcerated groin hernias: retrospective study. World J Surg. 2003;27:7413.

4. Butters M, Redecke J, Köninger J. Long-term results of a randomized clinical trial of Shouldice, Lichtenstein and transabdominal preperitoneal hernia repairs. $\mathrm{Br} \mathrm{J}$ Surg. 2007;94:562-5.

5. EU Hernia Trialists Collaboration. Repair of groin hernia with synthetic mesh: meta-analysis of randomized controlled trials. Ann Surg. 2002;235:322-32.

6. Bessa M, Katri K, Abdel-Salam W, Abdel-Baki N. Early results from the use of the Lichtenstein repair in the management of strangulated groin hernia. Hernia 2007;11:239-42.

7. Wysocki A, Kulawik J, Pozniczek M, Strzałka M. Is the Lichtenstein operation of strangulated groin hernia a safe procedure? World J Surg. 2006;30:2065-70.

8. Papaziogas B, Lazaridis CH, Makria J, Koutelidakis J, Patsas A, Grigoriou M, et al. Tension-free repair versus modified Bassini technique (Andrews technique) for strangulated inguinal hernia:a comparative study. Hernia 2005;9:156-9.

9. Grant AM. EU Hernia Trialists Collaboration. Open mesh versus non-mesh repair of groin hernia: metaanalysis of randomized trials based on individual patient data. Hernia 2002;6:130-6.

10. Álvarez J, Baldonedo R, García I, Suárez J, Álvarez P, Jorge J. Presentación y evolución clínica de las hernias externas incarceradas en pacientes adultos. Cir Esp. 2005;77:40-5.

11. Álvarez J, Baldonedo R, Bear I, Solís J, Álvarez P, Jorge $J$. Incarcerated groin hernias in adults: presentation and outcome. Hernia 2004;8:121-6.

12. Derici H, Unalp H, Bozdag A, Nazli O, Tansug T, Kamer E. Factors affecting morbidity and mortality in incarcerated abdominal wall. Hernias 2007;11:341-6.

13. Martínez-Serranoa MA, Pereiraa J, Sanchoa J, Salvansa 
S, Juncáb V, Segura M, y cols. Hernia estrangulada. ¿Todavía mortal en el siglo XXI? Cir Esp. 2008;83:199204.

14. Malone DL, Genuit T, Tracy JK, Gannon C, Napolitano LM. Surgical site infections: reanalysis of risk factors. J Surg Res. 2002;103:89-95.

15. Elsebae M, Nasr M, Said M. Tension-free repair versus Bassini technique for strangulated inguinal hernia: A controlled randomized study. Int J Surg. 2008;6:302-5.
16. Wysocki A, Poźniczek M, Krzywoń J, Strzalka M. Lichtenstein repair for incarcerated groin hernias. Eur J Surg. 2002;168:452-4.

17. Beltrán, M, Cruces K, Tapia T, Vicencio F. Resultados quirúrgicos de la hernioplastía de Lichtenstein de urgencia. Rev Chil Cir. 2006;58:359-64.

18. Ferzli G, Shapiro K, Chaudry G, Patel S. Laparoscopic extraperitoneal approach to acutely incarcerated inguinal hernia. Surg Endosc. 2004;18:228-31.

\section{Informe}

En primer lugar, deseo agradecer al directorio de la Sociedad de Cirujanos de Chile, el honor de haber sido designado Comentador del trabajo de ingreso del Dr. Francisco Venturelli M.

Antes de comentar el trabajo propiamente tal, quiero expresar algunas reflexiones sobre la patología herniaria, la que representa un 20 a $30 \%$ de la actividad quirúrgica del país.

Las modificaciones al esquema de trabajo de los servicios quirúrgicos del área pública, responsable del $80 \%$ de la población del país, como consecuencia de la ley de Garantías Explícitas de Salud (GES), conocidas como patologías AUGE, obliga a los jefes de servicios de cirugía a cumplir metas específicas, lo que origina la postergación de otras patologías NO AUGE, entre ellas la patología herniaria, originando el fenómeno de las listas de espera, que representa una demora en la resolución de estas enfermedades. Durante este período de espera los pacientes están expuestos a desarrollar complicaciones derivadas de la patología de base, como las que llevan a la cirugía de urgencia de la patología herniaria, motivo del presente trabajo.

Esta realidad, transforma la resolución de una patología de bajo riesgo, en una condición de riesgo vital.

Debemos, como Sociedad de Cirujanos, explicitar esta realidad y desarrollar las alternativas que permitan solucionar oportunamente este relevante problema de salud pública.

El trabajo presentado es una revisión retrospectiva de 148 pacientes, recopilados en un período de 5 años y representa un número significativo de casos. La metodología utilizada y el análisis estadístico son adecuadas.

Por tratarse de una serie de casos, las conclusiones de este trabajo sólo son aplicables a esta serie en particular, y su extrapolación no tiene certeza metodológica.

La edad promedio de la serie, de 66 años, impli- ca la posibilidad de tener comorbilidades médicas asociadas, las que se presentaron en cerca del $40 \%$ de la serie. Estas, en el análisis bivariado, representaron una asociación significativa de mayor riesgo de desarrollar una complicación postoperatoria para: obesidad, hipertensión arterial, diabetes mellitus y presentar ASA III y IV. En la regresión logística, sólo la hipertensión arterial representó un factor de riesgo.

Si categorizamos las variables estudiadas, como IMC para obesidad, podrían aparecer subgrupos de mayor riesgo, como los obesos mórbidos, lo que abre un interesante horizonte a futuros estudios.

La alta frecuencia de comorbilidades, significa enfrentar la resolución de la patología herniaria de urgencia, en pacientes con serios déficits sistémicos.

El aumento de riesgo de mortalidad se asoció en el análisis bivariado con: obesidad, hipertensión arterial, presentar ASA III y IV y presentar una hernia estrangulada.

En el análisis multivariado, sólo la estrangulación herniaria, representó un factor de riesgo.

Este último factor representa una demora significativa, entre el inicio del cuadro agudo y el momento de la cirugía, lo que puede deberse a tardanza en la consulta y/o en el diagnóstico.

En la presente serie se analizan en conjunto las diversas patologías, umbilical, inguinal, femoral e incisional, las que pueden tener diferentes conductas biológicas.

En nuestra experiencia, un grupo de alto riesgo, lo representa el de las mujeres mayores de 60 años, portadoras de hernia femoral complicada. En el grupo que requirió resección intestinal, la mortalidad se eleva a $28 \%$.

Esto también abre la posibilidad de estudiar las distintas conductas biológicas, de los distintos tipos de hernia.

El uso de material protésico en cirugía herniaria de urgencia, es un tema abierto a la discusión, no 
FACTORES DE RIESGO DE PRESENTAR UNA COMPLICACIÓN POSTOPERATORIA EN PACIENTES OPERADOS DE...

existiendo pautas consensuadas. En la presente serie, no representó un factor de riesgo para aumentar la morbimortalidad, lo que representa el buen criterio clínico utilizado para seleccionar los pacientes, a los cuales se les indicó malla, en urgencia.

La morbimortalidad de la presente serie es similar a la reportada en la literatura.

En la literatura hay una tendencia a intentar el uso de material protésico, en cirugía de urgencia, decisión que hasta ahora depende del criterio y la experiencia del cirujano. Trabajos como el presente, nos aportan datos objetivos, para seleccionar los pacientes con los menores riesgos de morbilidad asociados al empleo de material protésico en cirugía herniaria de urgencia.

Sra. Presidenta, en base a sus antecedentes y a la calidad del trabajo presentado, el Dr. Venturelli cumple con los requisitos para ser aceptado como socio titular, de la Sociedad de Cirujanos de Chile, y le deseamos una fructífera carrera.

Dr. Jaime Rappoport $S$. Santiago, 8 de septiembre de 2010. 